\title{
The effect of kinship on intergenerational cooperation: A lab experiment with three generations
}

\author{
José Alberto Molina ${ }^{1,2,3}$, Alfredo Ferrer ${ }^{3}$, J. Ignacio Giménez-Nadal ${ }^{1,3}$, Carlos \\ Gracia-Lazaro $^{3}$, Yamir Moreno ${ }^{3,4}$ and Angel Sanchez ${ }^{3,5,6}$
}

\begin{abstract}
In this paper, we analyze how kinship among family members affects intergenerational cooperation in a public good game. 165 individuals from 55 families, comprising three generations (youths, parents, and grandparents), play a public good game in three different treatments: one in which three members of the same family play each other (family), a second with the youth and two non-family members but preserving the previous generational structure (intergenerational), and a third in which three randomly-selected players play each other (random). We find that players contribute more to the public good when they play with other family members, than when they play with non-family members. This effect is present in all three generations, and is independent of the gender of the players. We also observe the significant result that older generations contribute more to the public good, relative to their children.
\end{abstract}

Keywords: Intergenerational cooperation, Evolutionary game theory, Public Goods game, Kinship, Social networks

JEL Codes: D03, D64, D70

\footnotetext{
${ }^{*}$ We are grateful to the IBERCIVIS Foundation, for volunteer recruitment. This paper has benefited from funding from the Spanish Ministry of Economics (Project ECO2012-34828). This paper was partially designed while Jose Alberto Molina was Visiting Fellow at the Department of Economics of Boston College (US), to which he would like to express his thanks for the hospitality and facilities provided. The authors declare no competing financial interests.

Correspondence to: J.A. Molina, Department of Economic Analysis, Faculty of Economics, C/ Gran Via 2, $3^{\text {rd }}$ floor, 50005 - Zaragoza, Spain. Tel.: +34976761818 Fax:+34976761996 email: jamolina@unizar.es.

${ }^{1}$ Departamento de Análisis Económico, Universidad de Zaragoza, ${ }^{2}$ Institute for the Study of Labor-IZA, Bonn, Germany, ${ }^{3}$ Instituto de Biocomputación y Física de Sistemas Complejos, Universidad de Zaragoza, ${ }^{4}$ Complex Networks and Systems Lagrange Lab, Institute for Scientific Interchange, Torino, Italy, ${ }^{5}$ Grupo Interdisciplinar de Sistemas Complejos (GISC), Departamento de Matemáticas, Universidad Carlos III de Madrid, ${ }^{6}$ Institute UC3M-BS for Financial Big Data, Universidad Carlos III de Madrid.
} 


\section{Introduction}

We analyze, using a public good game, how kinship among three generations of family members (youths, parents, and grandparents) affects their intergenerational cooperation. Although we begin by assuming that human behavior is essentially competitive, a number of relationships between individuals often show examples of cooperative and altruistic behaviors. Darwin (1859) perceived that this was a problem to an understanding and justification of his theory of evolution by natural selection: how can the existence of individuals cooperating for the benefit of others be explained, assuming, in many cases, a certain cost? The model that best explains the existence of individuals who cooperate is based on the family relationship, so that individuals within the family, who share genes, present the clearest examples of cooperative behavior motivated by generosity. This reasoning has been mathematically formalized through Hamilton's rule (Hamilton 1964) according to which cooperation between two individuals will take place if the benefit obtained, corrected by the kinship relationship between these individuals, exceeds the cost of giving or helping. This concept has been tried and tested empirically in the literature of the social sciences (see, among others, West et al. 2001; Oli 2003; Waibel et al. 2011).

Economists began to address cooperative behaviors from the time of Adam Smith (1759), who argued that interdependence between individuals provides positive utility measured in economic terms. Subsequently, Edgeworth (1881) justified this interdependence in terms of "social distance" between individuals and, more recently, the Nobel Laureate in Economics, Gary Becker $(1962,1974)$, established the generosity criterion guiding cooperation or transfer of resources from donor to recipient, with this approach contrasting with alternative hypotheses (Cox 1987), under which donors expect some kind of reciprocity from the receivers in a cooperative context.

The analysis of cooperation from the experimental sciences has a reference milestone in Nowak and May (1992), who developed a prisoner's dilemma to prove the survival of the cooperative agent in a complex network. Different versions of the prisoner's dilemma have been used to analyze cooperative behaviors, with a general result being that, in repeated dilemmas, the global cooperation level rapidly declines to values around 10\%, given the effect of free riders, with reciprocity (Trivers 1971), reputation (Novak and Sigmund 1998), punishment (Fehr and Gächter 2000; Sigmund et al. 2011) and the interplay of reputation with network rewiring (Cuesta et al. 2015), all being mechanisms to avoid the appearance of such free riders. 
In this context, it has also been observed in numerous public good experiments that many individuals contribute more to the public good than predicted by rationality and selfishness, with observed cooperation declining over time (see, for a survey, Chaudhuri 2011). One possible explanation is the assumption of conditional cooperation, according to which the individual propensity to cooperate depends upon the provisional cooperation of others (Fischbacher et al. 2001; Fischbacher and Gächter 2010). This assumption is considered to be a consequence of certain fairness preferences, such as altruism, commitment, reciprocity (Croson 2007) or warm-glow (Andreoni 1990).

Prior research on cooperation using experiments has found mixed results from a gender perspective, although recent evidence appears to point toward women being more cooperative than men (Molina et al. 2013). With respect to age, experimental research has shown that younger children are less altruistic (Fehr et al. 2008: Fehr et al. 2011). However, while the repeated prisoner's dilemma has been used extensively in experiments with adults (Blake et al. 2015), experiments with children remain rare, with the exception of Blake et al. (2015), who use pre-adolescent children (mean age 11.6 years).

Little is known about how cooperative behavior changes across generations. Two papers have included subjects of different ages who were involved in the same experimental set-up to test their cooperativeness (Charness and Villeval 2009; and Gutiérrez-Roig et al. 2014). In the first, the authors conducted experiments with employees of two French firms using junior (under 30) and senior (over 50) subjects, and in a conventional laboratory set-up with students and retirees, with their main result being that seniors were more cooperative than juniors. In the second, volunteers of different ages were placed in a group with other players of similar ages to play n-player prisoner's dilemmas, with the main finding being that the behavior is quite similar in age groups between 18 and 65 years old; teenagers' behavior is less predictable, and the elderly are more cooperative. In both papers, whose results are in agreement with the prior literature, individuals of different ages play within the same generation, but, to the best of our knowledge, only Peters et al. (2005), Bauer et al. (2014), and Porter and Adams (2015) focus on intergenerational issues, on the basis of the well-established evidence that the preferences of one generation can be affected by the preferences of the preceding one, in such a way that parental background has been found to be related to the fundamental life outcomes of children. 
Peters et al. (2005) place parents and children in a laboratory to participate in a public good experiment, with the main result being that parents may behave more altruistically than do their children in a family setting. Bauer et al. (2014) uses individuals of two generations to prove, among other results, that children of parents with a lower level of education are less altruistic. Porter and Adams (2015) study the motivations behind transfers between two generations (adult children and their parents) in an experimental setting, in such a way that the subjects play a series of dictator games in the laboratory, with results indicating that a greater proclivity for giving appears when parents, rather than strangers, are the recipients of transfers.

Against this background, the novelty of our research is that we analyze how kinship among family members affects the intergenerational behaviors between three generations (youths, parents, and grandparents) when individuals of different generations play a public good game (http://www.ibercivis.es/projects/colabora-con-laciencia-en-familia/). Volunteers aged between 17 and 19 were recruited to participate in an experiment where the only information given a-priori was that the volunteers will play with one of their parents and one of their grandparents. After presenting the laboratory results, we use data computed from the experiment to estimate econometric models that take into account the unobserved heterogeneity of individuals, since there may be certain unobserved factors at the individual level that are correlated with the level of contribution, which would bias the results. For instance, past personal experience, the mood on the day of the experiment, or personal attitudes towards justice, equity, and confidence, may all condition the decisions of individuals in our experiment. Thus, we first estimate a random-effects linear model for the pooled data, observing that the behavior is different across groups of individuals and, consequently, we have also estimated the model considering the groups of youths, parents, and grandparents separately. Additionally, and in order to take advantage of the panel structure of the data, two types of panel data estimators are estimated: the random-effects and fixedeffects estimators.

Our results indicate that the level of cooperation of the youths is significantly lower than that of the parents and the grandparents. All the age groups cooperate more when playing with relatives, with this trend being more evident for the youths and the parents than for the grandparents. Thus, kinship appears to have a positive relationship with contribution to the common good, with this contribution being strongly 
conditioned by both the own previous action and the last observed contributions. We have also developed an empirical strategy to take into account the unobserved heterogeneity of individuals, with results indicating clear differences in behavior across our three generations. Finally, panel estimations confirm that playing with family members increases the contribution of players to the public good, thus indicating that kinship has a positive effect on the contribution of the players.

Our contribution to the literature is twofold. First, we design and carry out an intergenerational experiment, with three generations, in order to evaluate how kinship among family members affects intergenerational behaviors. Each volunteer participated in three different 3-player games, corresponding to three different treatments: one in which three members of the same family played each other, a second with the youth and two non-family members, but preserving the previous generational structure, and a third in which three randomly-selected players played each other. Second, we develop an exhaustive econometric analysis to take into account the unobserved heterogeneity of individuals by estimating random-effects linear models for the pooled data, as well as for the three age groups separately, and we estimate the random-effects and fixedeffects models to take advantage of the panel structure of the data.

The rest of the paper is organized as follows. Section 2 describes the intergenerational public good experiment. Section 3 presents the lab-in-the-field results. Section 4 shows the empirical strategy and the estimation results, and Section 5 sets out our main conclusions.

\section{Intergenerational public good experiment}

\subsection{Design}

We performed two series of lab-in-the-field experiments involving 165 volunteers: 55 volunteers aged between 17 and 19 years old, one of their parents, and one of their grandparents. Each volunteer participated in three different 3-player games, corresponding to three different treatments: (a) one in which three members of the same family (i.e., youths, parents, and grandparents) played each other (family treatment), (b) a second with the youth and two non-family members but preserving the previous generational structure (intergenerational treatment), and (c) a third in which three randomly-selected players played each other (random treatment) (see the Methods section for further details). 
In the public good game, participants played a given number of rounds, not known a priori by the players, although the number of rounds was set at 10 in each treatment by the research group, and thus we have 30 games per individual. In each round, participants had to decide how many monetary units (hereafter, Experimental Currency Units, ECUs) they wanted to "invest" (from 0 to 10) in the common fund, and they also had information on how many units the other players had invested in the previous round (except in the first round of each treatment). In each round, the sum of the contributions made by all 3 players was calculated, and the total contribution was multiplied by a factor of 1.5 and then shared equally by the 3 players. The obtained payoff in each round was the sum of this share, plus the ECUs held back, i.e., not invested in the common fund. This payoff could not be used in subsequent rounds. At the end of the experiment, each player received the sum of the payoffs corresponding to all rounds of the three treatments in which he/she participated. ECUs were converted into real monetary units, euros $(€)$, according to the proportion derived from the total cost of the experiment $(1,720 €)$. Typical earnings ranged from $8 €$ to $12 €$, including a $5 €$ attendance fee.

In the treatment including all family members, the game is played by three generations of the same family: youth, parent, and grandparent (with independence of the gender and age of the players). In the treatment including non-family members, but maintaining the structure of the population, there is still one youth, one parent, and one grandparent, but they are not related. In the third treatment, where the assignment is random, each player is matched randomly, independent of the relationship and generation of the players. These three treatments allow us to isolate the effect of kinship from other effects, which includes the generation (prior research has found that cooperation increases with age; see Peters et al. 2015; Porter and Adams, 2015; Gutiérrez-Roig et al., 2014) and gender (prior research has found that cooperation is more common in women than in men; see Molina et al. 2013). Using the three treatments, if kinship across players has any effect, we would expect to find differences in the behavior of players between the family treatment and the intergenerational and random treatments. Furthermore, if we find no differences in the behavior of participants between the intergenerational and random treatments, this may be interpreted as that the structure of the game has no effect on the level of contribution of the participants. 


\subsection{Methods}

As a fundamental ethics statement, the anonymity of all participants in the experiments reported was always preserved (in accordance with the Spanish Law on Personal Data Protection) by assigning them, randomly, a username that would identify them in the system. No association was ever made between their real names and the results. As is standard in socio-economic experiments, no ethical concerns are involved, other than preserving the anonymity of participants.

Volunteers have been recruited from the general population of the city of Zaragoza (Spain). The selection process consisted of attracting sets of three volunteers of different generations of the same family, by filling-in an on-line form. Applications were open to any set of three relatives meeting the above conditions, regardless of their social status or other demographic variables. The total number of participants was 165 , and the 55 families were distributed in two groups. While the first group ( $\mathrm{n}=34$ families) played treatments in a given order (i.e., family, intergenerational, random), the second group ( $\mathrm{n}=21$ families) played treatments in the opposite order (i.e., random, intergenerational, family). The Appendix shows the tutorial used in the experiment, and the support staff were not informed about the scope of the experiment.

Detailed instructions were read aloud at the beginning of the experiment and provided in a text that participants read and accepted before starting the experiment. After each session, the participants were informed of the amount of money they had won and immediately paid. The experiments were performed on a web-based platform and results were gathered in a database for further analysis. Individuals played anonymously and could not interact during the experiment. Participant anonymity was always preserved and non-personal data was collected.

\section{Lab-in-the field results}

Figure 1 shows the average contribution per round, computed over all the volunteers of the same generation. Different symbols represent different generations (a red circle for the youth, a blue square for the parent, and a green triangle for the grandparent) while different panels correspond to different treatments (left panel for family treatment, center panel for inter-generational, and right panel for random). As shown, regardless of 
the nature of the partners and the generation membership, the average contributions to the public good remain roughly constant over time.

(Figure 1 about here)

Figure 2 represents the contributions to the common fund, averaged over all the rounds. Panel A of Figure 2 shows the results per generation (i.e., youths, parents, grandparents) aggregated over all the treatments. As can be seen, the level of cooperation of the youths (5.05) is significantly lower than that of the parents (6.21) and the grandparents (5.95). Panel B of Figure 2 shows the average contributions per treatment (i.e., family, inter-generational, random), showing that, when an individual played with other members of the family, more is invested in the public good (6.77) than when interacting with strangers, whether they interact with members of different generations (5.27) or random participants (5.17). There was no significant difference between these two latter cases. The absence of significant differences in contributions between inter-generational and random treatments shows that the generational structure, by itself, does not have a significant impact on the common-good contributions. Finally, panel $\mathrm{C}$ of Figure 2 shows the contributions per generation and per treatment, averaged over all rounds of a given treatment and generation. It can be observed that kinship promotes cooperation across all generations, given that, for all age groups, the contributions to the public good when interacting with relatives are higher than when playing with strangers. Although all the age groups cooperate more when playing with relatives, this trend is more evident for the youths and the parents than for the grandparents, which suggests that grandparents are less influenced by kinship with respect to the common-good contributions

(Figure 2 about here)

In order to study the dynamics of individual contributions, we have examined the variation of contributions (i.e., the increase in individual contributions) as a function of the difference between the own and partners' last action; that is, we considered the possibility that players react to the contributions they observed in the previous round, by assuming that players have a one-step memory. Figure 3 shows the probability, from white for $\mathrm{p}=0$ to red for $\mathrm{p}=1$, of the contribution increment (y-axis, the difference between the contribution of an individual in a given round and his/her contribution in the previous round) as a function of the difference between the other players' contributions and the own contribution in the previous round (x-axis), for the different 
generation groups in different panels: youths in panel A, parents in panel B, and grandparents in panel C. As can be seen, there is a very strong positive dependence of the contribution increment on the difference between own and partners' last action. This dependence is more pronounced in the group of parents. To more fully appreciate this dependence, panel A of Figure 4 shows the probability of increasing the own contribution to the public good as a function of the difference between the other players' averaged contribution and the own contribution in the previous round, aggregated over all the treatments (family, inter-generational, random), for the different generation groups (youths, parents, and grandparents). As shown, there is a positive correlation between the contribution increments and the difference in contributions. Additionally, panel B of Figure 4 represents the probability of decreasing the own contribution versus the difference between other players' and own contributions in the previous round. As a corollary, Figures 3 and 4 show that the contribution to the common good is strongly conditioned by both the player's previous action and the last observed contributions.

(Figure 3 about here)

(Figure 4 about here)

Table 1 shows average contributions of players, and players' characteristics. Column (1) shows average values for all the sample, and Columns (2), (3), and (4) show average values for youths, adults, and grandparents, respectively. We observe that the average contribution to the public good is 5.73 . However, when we look at the different groups of players, we observe that the average contributions of youths, adults, and grandparents are 5.05, 6.21, and 5.95, respectively. Furthermore, t-type tests of equality of means show that the average contribution varies across the three groups, as differences are statistically significant at the $99 \%$ confidence level.

(Table 1 about here)

The second panel of Table 1 analyzes the average contribution to the public good, by treatment. We observe that average contributions in the family (Column 2), generational (Column 3), and random (Column 4) treatments are 6.77, 5.27, and 5.17, respectively. Furthermore, t-type tests of equality of means show that the average contribution varies between the family treatment and the other two treatments, as differences are statistically significant at the $99 \%$ confidence level. Thus, differences in the average contribution between the family and the generational treatments, on the one 
hand, and the family and the random treatments, on the other, are 1.50 and 1.59, respectively. We therefore conclude that kinship across parents has a causal effect on the contribution of players.

Figure 5 shows the mean and confidence intervals at the $95 \%$ confidence level of the contribution of players by generation, treatment, and round number. The first graph shows the average contribution for youths, the second the average contribution for parents, and the third the average contribution for grandparents. We observe that, for the three generations, the contribution made by the players in the family treatment is comparatively higher than the contribution made in the generational and random treatments, for all rounds. Furthermore, the confidence intervals of the family treatment do not intersect with the confidence intervals of the generational and random treatments, indicating that the level of contribution made in the family treatment by the three groups of players is higher than the contribution made in the other two treatments. ${ }^{2}$ Furthermore, we cannot conclude that the level of contribution differs between the generational and random treatments, as their confidence intervals intersect in the three groups. Thus, we observe that kinship appears to have a positive relationship to contributions, as for all players the contribution increases when they play with their relatives, in comparison with playing with other, unknown players, and the fact that we find no statistically significant differences between the generational and random treatment indicates that the population structure (i.e., the number of players of each generation) does not appear to affect the level of contribution.

(Figure 5)

One third (33\%) of the players are male, and the average age is 48.09 years. By group of players, $35 \%, 38 \%$, and $27 \%$ of youths, parents, and grandparents are male, with an average age of $17.44,50.62$, and 76.22 , respectively. Furthermore, $38 \%$ of the players pertain to the second group. Here we must acknowledge that, given that the order of treatments is reversed in the second group, compared to the first group, this may affect the results.

To disentangle this possible effect, we have analyzed in Table 2 the average contribution to the public good, by group and treatment. The average contributions in the family, generational, and random treatments in the first group are 6.01, 4.89, and

\footnotetext{
${ }^{2}$ The only exception is round 4 for the group of grandparents
} 
4.91, respectively, while the average contributions in the family, generational, and random treatments in the second group are 7.99, 5.88, and 5.60. Thus, the differences in the average contribution between the two groups are $1.98,0.99$, and 0.69 for the family, generational, and random treatments, respectively, with such differences being statistically significant at standard levels. These results indicate that the contributions of the second group are comparatively higher than in the first group, independent of the treatment, and such differences cannot be attributed to the difference in the order of treatments. Similar conclusions can be drawn when we look at the average contribution by group, treatment, and round. Figure 6 shows the mean and confidence intervals at the $95 \%$ confidence level of the contribution of players by group, treatment, and round. We observe that, in almost all cases, there is a difference in the level of contribution favoring the second group, with these differences being statistically significant at standard levels (that is, confidence intervals do not intersect).

(Table 2 about here)

(Figure 6 about here)

\section{Empirical strategy and estimation results}

We estimate models that take into account the unobserved heterogeneity of individuals, since there may be certain unobserved factors at the individual level that are correlated with the level of contribution, which would bias the results. For instance, past personal experience, the mood on the day of the experiment, or personal attitudes towards justice, equity, and confidence, all may condition the decisions of individuals in our experiment. Thus, we estimate a random-effects linear model to control for the unobserved heterogeneity of individuals, using the following equation: ${ }^{3}$

$$
\mathrm{C}_{\mathrm{ij}}=\alpha_{i}+\beta_{1} \text { Family }_{i j}+\beta_{2} \text { Generational }_{i j}+\delta \mathrm{X}_{i}+\varepsilon_{\mathrm{ij}}
$$

where $C_{i j}$ represents the decision (contribution) by individual " $\mathrm{i}$ " in round " $\mathrm{j}$ ", and $\alpha_{i}$ represent the individual effect. The dependent variable is a variable that measures the amount given by individual " $\mathrm{i}$ " in round " $\mathrm{j}$ " to the common fund. We include two dummy variables to indicate whether player " $\mathrm{i}$ " is playing in the family or generational

\footnotetext{
${ }^{3}$ The time variation needed to estimate a panel data model is given by the fact that respondents played more than one round during each phase.
} 
treatment, and thus the reference group is players in the random treatment. According to our hypothesis (that kinship increases the level of contributions), we should obtain that $\beta_{1}>0$. The vector $X_{i}$ includes the demographic characteristics of participant "i", such as gender $(1=$ male, $0=$ female $)$, age of the player and its square, two dummy variables to indicate whether the player pertains to the group of grandparents or parents (ref:: youths), and a dummy variable to control for whether the player pertains to the second group (1) or not (0). We also control for the round number to account for possible learning effects. $\varepsilon_{i j}$ is a random variable (standard error) that represents unmeasured factors, capturing all the factors that may affect those participant decisions for which we do not have information, and we assume that $\varepsilon_{\mathrm{ijt}} \sim N\left(0, \sigma^{2}\right)$.

We estimate several models, according to several dimensions. The first refers to the generation the players belong to. Even though we estimate Equation (1) for the pooled data, we have observed that the level of contribution differs across the generations, which may imply that behavior is different across groups of individuals. Thus, we also estimate Equation (1) considering the group of youths, parents, and grandparents separately. Second, and in order to take advantage of the panel structure of the data, two types of panel data estimators are estimated: the random-effects (RE) and fixed-effects (FE) estimators.

Table 3 shows the results of estimating Equation (1) for the sample of players. Columns (1), (3), (5) and (7) show the results of using the RE estimator for the pooled sample (all), the youths, the parents, and the grandparents, respectively. Columns (2), (4), (6) and (8) show the results of using the FE estimator for the pooled sample, the youths, the parents, and the grandparents, respectively. We observe positive and statistically significant coefficients for the variable representing the family game, for the pooled sample, and all the subsamples, and using both the RE and FE estimator. For the pooled sample, we observe that, in comparison with the random treatment, playing in the family treatment increases the average contribution by 1.72 monetary units. In the case of the different subsamples, we observe that the greater effect is found for the group of youths, followed by parents, and then the group of grandparents, as playing in the family treatment increases the average contribution by 2.46, 1.70 and 1.01 monetary units, respectively. If we now focus on the generational treatment, in comparison with the random treatment, we observe that the coefficients of the dummy variable are not statistically significant. Thus, we find that, in comparison with the random and 
generational treatment, playing with family members increases the contribution of players to the public good, indicating that kinship has a positive effect on the contribution of the players.

(Table 3 about here)

\section{Conclusions}

It is well-established in two-generation studies that parental background is related to the fundamental life outcomes of children. However, no experimental evidence, to the best of our knowledge, has been provided for the level of cooperation among three generations. To bridge this gap, we have developed in this paper a public good experiment with three generations (youths, parents, and grandparents) with the participation of 165 volunteers (55 aged between 17 and 19, one of their parents, and one of their grandparents).

Our results indicate that the level of cooperation of the youths is significantly lower than that of the parents and the grandparents. All the age groups cooperate more when playing with relatives, with this trend being more evident for the youths and the parents than for the grandparents. Thus, kinship appears to have a positive relationship to contributions to the common good, with these contributions being strongly conditioned by both the own previous action and the last observed contributions.

We have also developed an empirical strategy to take into account the unobserved heterogeneity of individuals, by estimating a random-effects linear model, with results indicating clear differences in behavior across our three generations. Additionally, to take advantage of the panel structure of the data, we have also estimated a random-effects and a fixed-effects model, with results confirming that playing with family members increases the contribution of players to the public good, thus indicating that kinship has a positive effect on the contribution of the players.

\section{REFERENCES}

Andreoni, J. (1990). Impure altruism and donations to public goods: a theory of warmglow giving. Economic Journal, 100, 464-477. 
Bauer, M., Chytilová, J., and Pertold-Gebicka, B. (2014). Parental background and other-regarding preferences in children. Experimental Economics, 17, 24-46.

Becker, G.S. (1962). Investment in human capital: a theoretical analysis. Journal of Political Economy, 70, 9-49.

Becker, G.S. (1974). A Treatise on the Family. Harvard University Press.

Burlando, R.M., and Guala, F. (2005). Heterogeneous agents in public godos experiments. Experimental Economics, 8, 35-54.

Blake, P.R., Rand, d.G. Tingley, D., and Warmeken, F. (2015). The shadow of the future promotes cooperation in a repeated prisoner's dilemma for children. Scientific Reports, 5, 14559.

Cox, D. (1987). Motives for Private Transfers. Journal of Political Economy, 95, 508546.

Croson, R.T.A. (2007). Theories of commitment, altruism and reciprocity: evidence from linear public goods games. Economic Inquiry, 45, 199-216.

Cuesta, J.A., García-Lázaro, C., Ferrer, A., Moreno, Y., and Sánchez, A. (2015). Reputation drives cooperative behaviour and network formation in human groups. Scientific Reports, 5, 7843.

Chaudhuri, A. (2011). Sustaining cooperation in laboratory public goods experiments: a selective survey of the literature. Experimental Economics, 14, 47-83.

Darwin, C. (1859). On the Origin of Species by Means of Natural Selection or the Prevervation of Favored Races in the Struggle for Life. John Murray, London.

Edgeworth, F. (1881). Mathematical Psychics: An Esssay of the Application of Mathematics to the Moral Sciences. C. Kegan Paul and Co. London.

Fehr, E., and Gächter, S. (2000). Cooperation and punishment in public goods experiments. American Economic Review, 90, 980-994.

Fischbacher, U., and Gächter, S. (2010). Social preferences, beliefs, and the dynamics of free riding in public goods experiments. American Economic Review, 100, 541-556.

Fischbacher, U., Gächter, S., and Fehr, E. (2001). Are people conditionally cooperative? Evidence from a public goods experiments. Economics Letters, 71, 397-404. 
Gutiérrez-Roig, M., García-Lázaro, C., Perelló, J., Moreno, Y., and Sánchez, A. (2014). Transition from reciprocal cooperation to persistent behaviour in social dilemmas at the end of adolescence. Nature Communications, 5, 4362.

Hamilton, W.D. (1964). The genetical evolution of social behaviour. Journal of Theoretical Biology, 7, 1-52.

Molina, J.A., Giménez-Nadal, J.I., Cuesta, J.A., García Lázaro, C., Moreno, Y., and Sanchez, A. (2013). Gender differences in cooperation: experimental evidence on high school students. Plos One, 8 (12), e83700.

Nowak, M.A., and May, R.M. (1992). Evolutionary games and spatial chaos. Nature, $359,826-829$.

Nowak, M.A., and Sigmund, K. (1998). Evolution of indirect reciprocity by image scoring. Nature, 393, 573-577.

Oli, M.K. (2003). Hamilton goes empirical: estimation of inclusive fitness from lifehistory data. Proceedings of the Royal Society Biological B, 270, 307-311.

Peters, H.E., Ünür, A.S., Clark, J., and Schulze, W.D. (2004). Free-riding and the provision of public goods in the family: a laboratory experiment. International Economic Review, 45, 283-299.

Porter, M, and Adams, A. (2015). For love or reward? Characterising preferences for giving to parents in an experimental setting. The Economic Journal. DOI: 10.1111/econj.12248.

Sigmund, K, Hauert, C., and Novak, M.A. (2001). Reward and punishment. Proc Nat Aca. Science. 98, 10757-10762.

Smith, A. (1759). The Theory of Moral Sentiments. Printed for A. Millar, in the Strand; and A. Kincaid and J. Bell, in Edinburgh.

Trivers, R.L. (1971). The evolution of reciprocal altruism. Quarterly Review of Biology, $46,35-57$.

Waibel, M., Floreano, D., and Keller, L. (2011). A Quantitative test of Hamilton's rule for the Evolution of Altruism. PloS Biology, 9 (5), 1-7.

West, S.A., Murray, M.G., Machado, C.A., Griffin, A.S., and Herre, E.A, (2011). Testing Hamilton's rule with competition between relatives. Nature, 409, 510-513. 
A

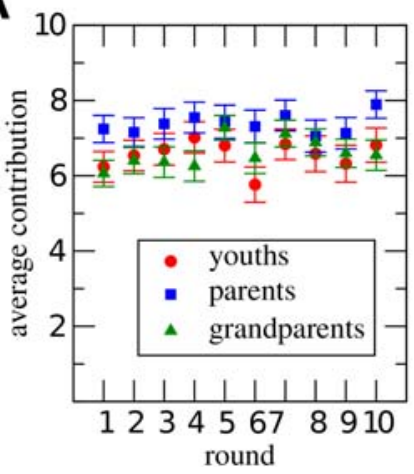

B

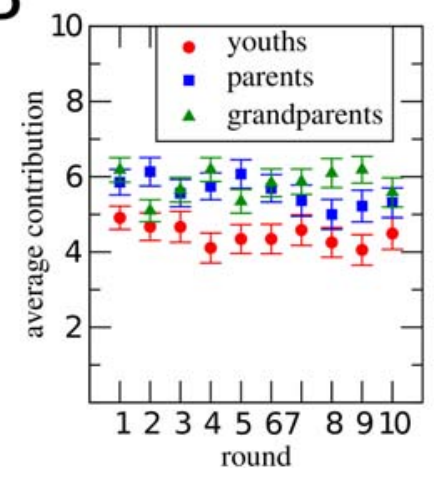

C

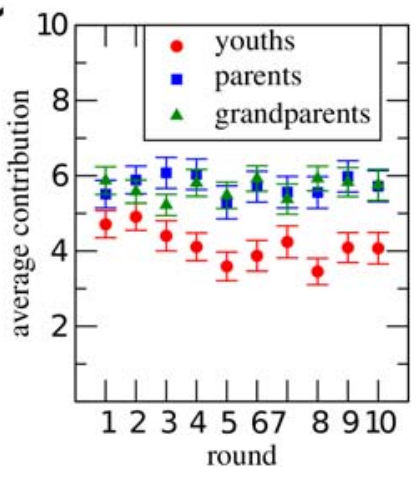

Figure 1 Contributions remain constant over time. Level of cooperation (average contribution) per round, averaged over all the participants for the same generation: youths (red circles), parents (blue squares) and grandparents (green triangles). Panel A represents the treatment where each participant faces a public goods game with two other members of the family from different generations, panel B stands for the treatment where unrelated participants from different generations face each other, and panel $\mathrm{C}$ shows the treatment where participants were matched at random in groups of three. As shown, averaged contributions remain roughly constant over time. Error bars represent the Standard Error of the Mean. 

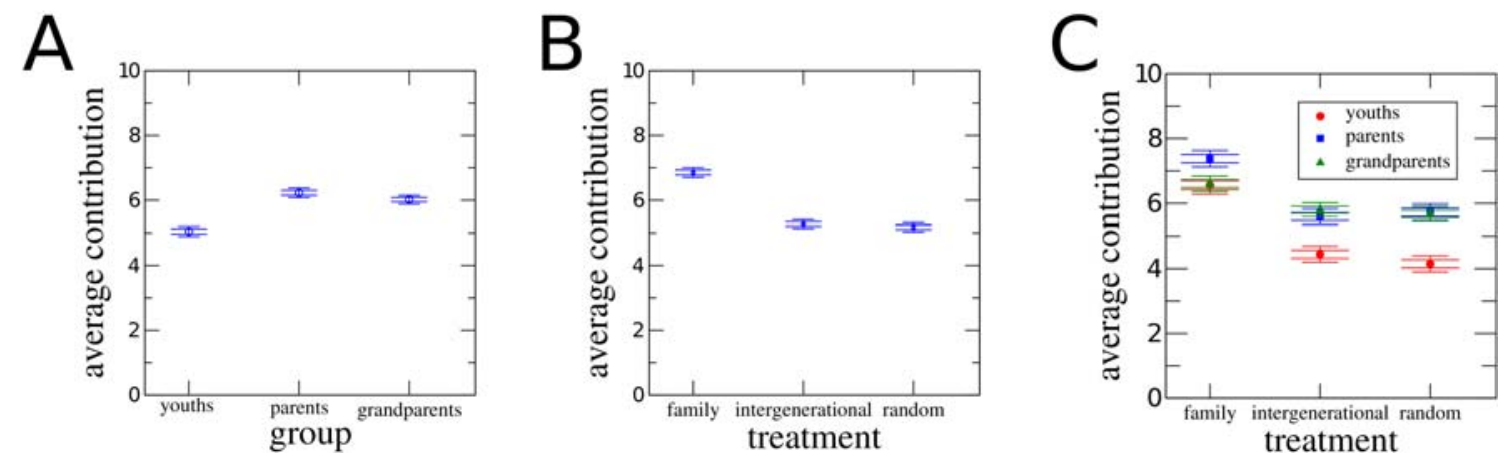

Figure 2 Youths contribute less than parents and grandparents, while all the generations cooperate more when interacting with relatives. Level of cooperation (average contribution) averaged over all the rounds. In panel A, results from different treatments (i.e., family, intergenerational, random) have been aggregated, showing the averaged contributions for each generation. As shown, youths exhibit a lower level of cooperation, while parents and grandparents contribute more. In panel B, results from different generations have been aggregated, showing the averaged contributions for each treatment. When an individual interacts with members of the family, that individual contributes more to the public good than when playing with strangers, regardless of the knowledge of the generation. Finally, panel $\mathrm{C}$ shows averaged contributions per generation and per treatment. Although all the three generations exhibit higher levels of cooperation when playing with relatives, this trend is less pronounced for the grandparents. Error bars represent the Standard Error of the Mean. 
A

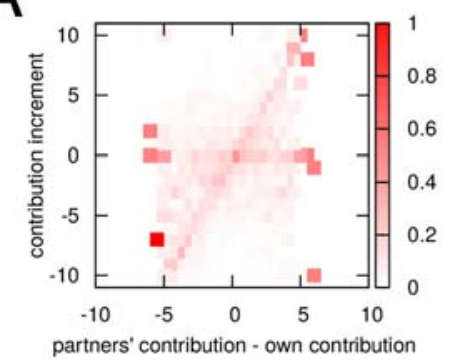

B

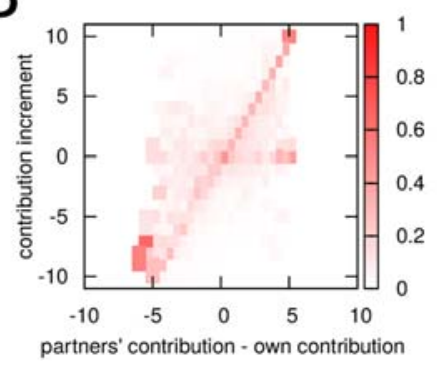

C

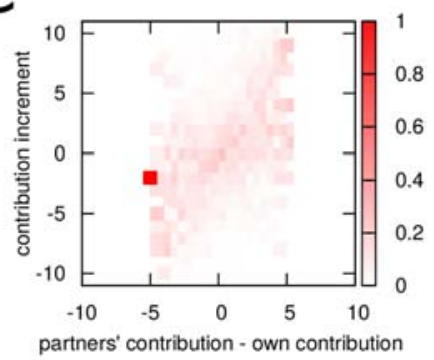

Figure 3 Contributions depend on own and partners' last action. Probability (color code, from white for $\mathrm{p}=0$ to red for $\mathrm{p}=1$ ) of own contribution increment (y-axis) as a function of the difference between averaged partners' contribution and own contribution in the last round. As shown in the three panels (A: youths, B: parents, C: grandparents), the difference between own and partners' last action strongly conditions the contribution increment. 

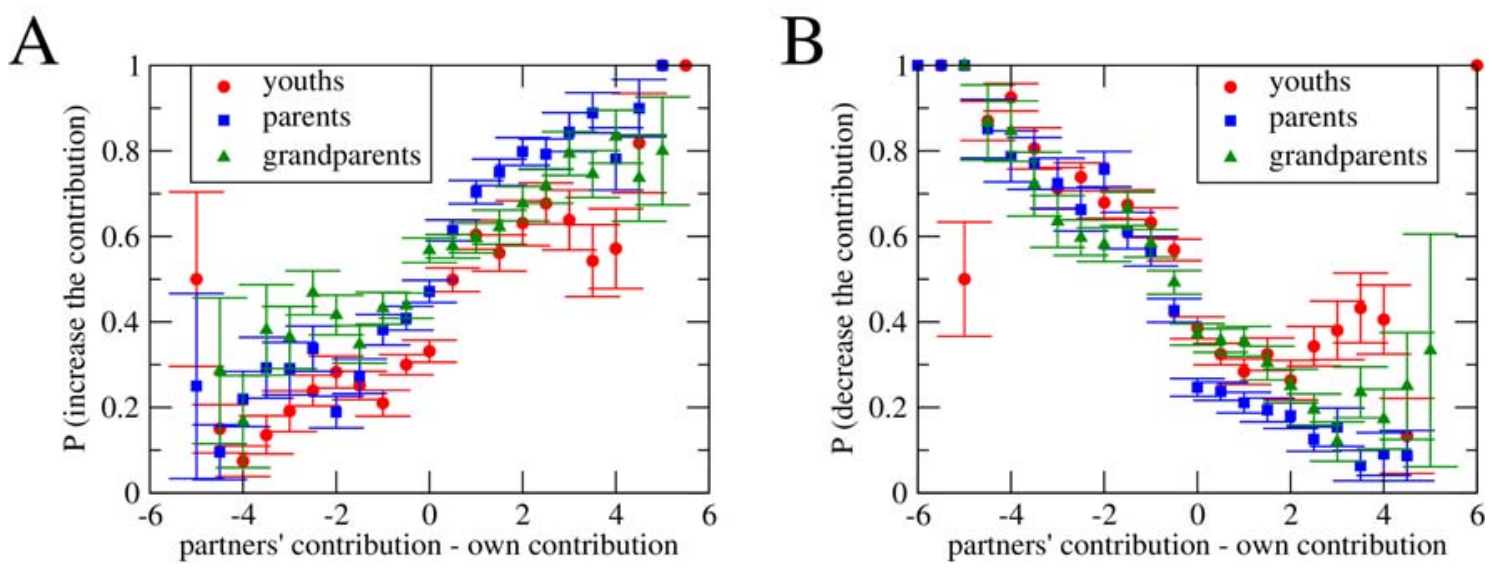

Figure 4 Contributions increment positively correlate with past contributions differences. Panel A (B) represents the probability to increase (decrease) the own contribution to the public fund as a function of the difference between averaged partners' contribution and own contribution in the last round. In both panels, results from different treatments (i.e., family, intergenerational, random) have been aggregated. Error bars represent the Standard Error of the Mean. 

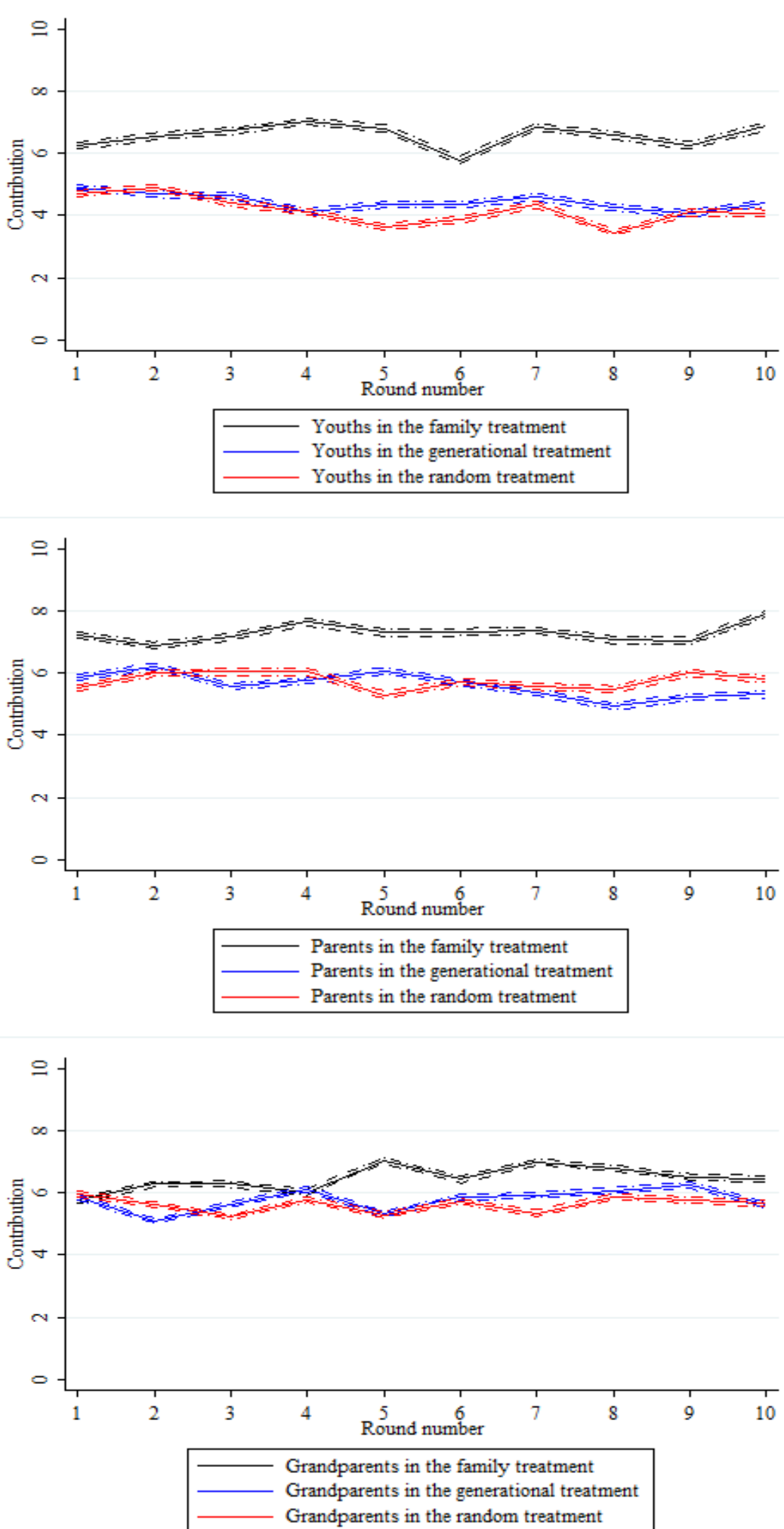

Figure 5 Average contribution by generation, treatment, and round. Contribution measures the contribution in each round to the public good, on a scale from 0 to 10 . Confidence intervals are calculated as $\bar{X} \pm 1.96^{*} S E$, where $\bar{X}$ represents the mean value, and SE represent the Standard Error. 

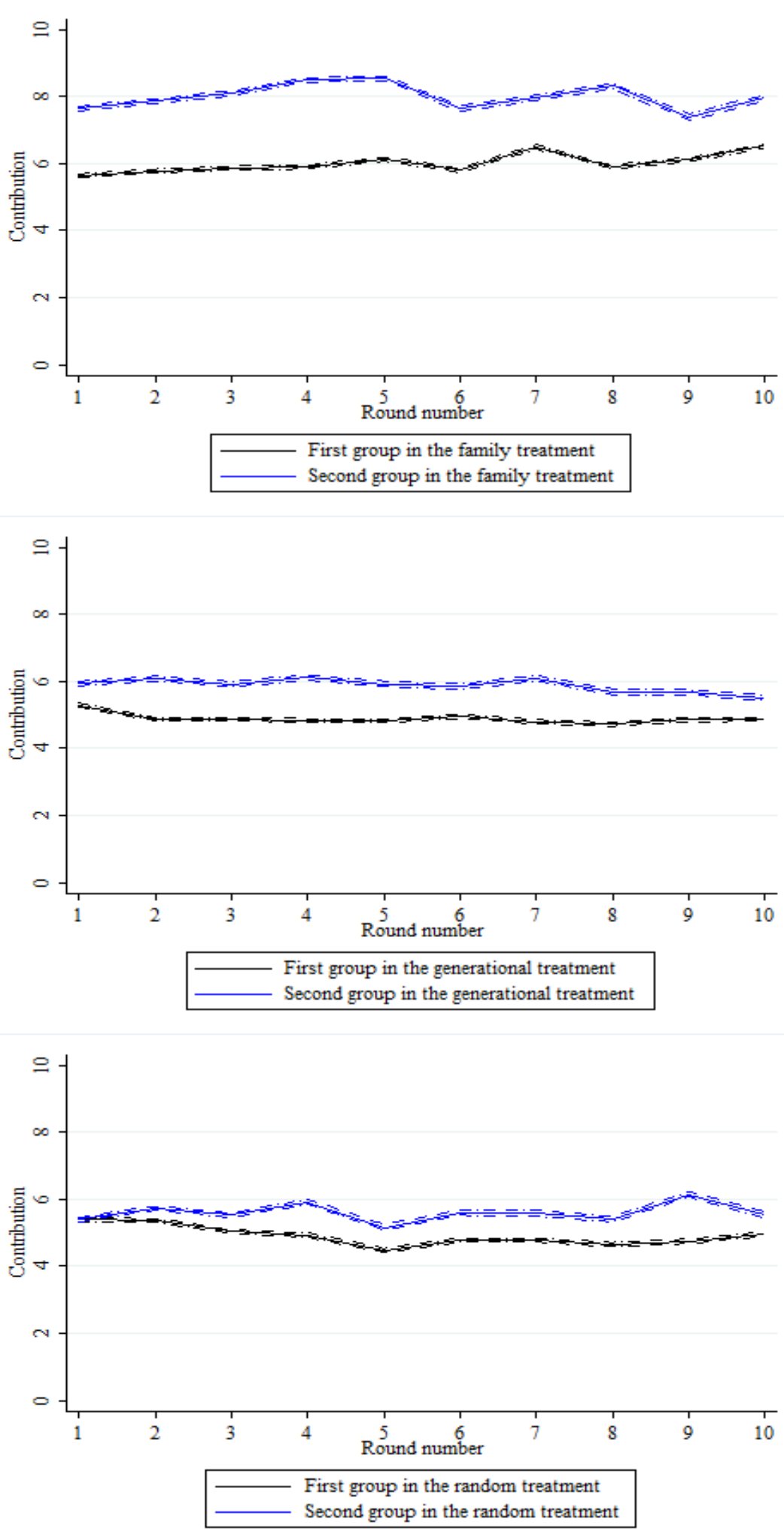

Figure 6 Average contribution by session, treatment, and round. Contribution measures the contribution in each round to the public good, on a scale from 0 to 10 . Confidence intervals are calculated as $\bar{X} \pm 1.96 * S E$, where $\bar{X}$ represents the mean value, and SE represent the Standard Error. 
Table 1 Sum Stats

\begin{tabular}{|c|c|c|c|c|}
\hline & $\begin{array}{l}\text { (1) } \\
\text { All }\end{array}$ & $\begin{array}{c}\text { (2) } \\
\text { Youths }\end{array}$ & $\begin{array}{c}\text { (3) } \\
\text { Parents }\end{array}$ & $\begin{array}{c}\text { (4) } \\
\text { Grandparents }\end{array}$ \\
\hline Contribution & $\begin{array}{c}5.73 \\
(0.04)\end{array}$ & $\begin{array}{c}5.05 \\
(0.05)\end{array}$ & $\begin{array}{c}6.21 \\
(0.04)\end{array}$ & $\begin{array}{l}5.95 \\
(0.04)\end{array}$ \\
\hline Male & $\begin{array}{c}0.33 \\
(0.01)\end{array}$ & $\begin{array}{c}0.35 \\
(0.01)\end{array}$ & $\begin{array}{c}0.38 \\
(0.01)\end{array}$ & $\begin{array}{c}0.27 \\
(0.01)\end{array}$ \\
\hline Age & $\begin{array}{l}48.09 \\
(0.36)\end{array}$ & $\begin{array}{l}17.44 \\
(0.01)\end{array}$ & $\begin{array}{l}50.62 \\
(0.10)\end{array}$ & $\begin{array}{l}76.22 \\
(0.15)\end{array}$ \\
\hline Second group & $\begin{array}{c}0.38 \\
(0.01)\end{array}$ & $\begin{array}{c}0.38 \\
(0.01)\end{array}$ & $\begin{array}{c}0.38 \\
(0.01)\end{array}$ & $\begin{array}{c}0.38 \\
(0.01)\end{array}$ \\
\hline $\mathrm{N}$ observations & 4950 & 1650 & 1650 & 1650 \\
\hline $\mathrm{N}$ participants & 165 & 55 & 55 & 55 \\
\hline $\begin{array}{l}\text { Diff Youths-Parents } \\
\text { P-value diff }\end{array}$ & \multicolumn{4}{|c|}{$\begin{array}{c}-1.16 \\
(<0.01)\end{array}$} \\
\hline $\begin{array}{l}\text { Diff Youths-Grandparents } \\
\text { P-value diff }\end{array}$ & \multicolumn{4}{|c|}{$\begin{array}{c}-0.9 \\
(<0.01)\end{array}$} \\
\hline $\begin{array}{l}\text { Diff Parents-Grandparents } \\
\text { P-value diff }\end{array}$ & \multicolumn{4}{|c|}{$\begin{array}{c}0.26 \\
(0.01)\end{array}$} \\
\hline & & Familly & Generations & Random \\
\hline Contribution & & $\begin{array}{c}6.77 \\
(0.04)\end{array}$ & $\begin{array}{c}5.27 \\
(0.04)\end{array}$ & $\begin{array}{l}5.17 \\
(0.04)\end{array}$ \\
\hline $\begin{array}{l}\text { Diff family-generations } \\
\text { P-value diff }\end{array}$ & \multicolumn{4}{|c|}{$\begin{array}{c}1.50 \\
(<0.01)\end{array}$} \\
\hline $\begin{array}{l}\text { Diff family-random } \\
\text { P-value diff }\end{array}$ & \multicolumn{4}{|c|}{$\begin{array}{c}1.59 \\
(<0.01)\end{array}$} \\
\hline $\begin{array}{l}\text { Diff generations-random } \\
\text { P-value diff }\end{array}$ & \multicolumn{4}{|c|}{$\begin{array}{c}0.09 \\
(0.35)\end{array}$} \\
\hline
\end{tabular}

Notes: Standard Errors in parenthesis. Contribution measures the contribution in each round to the public good, on a scale from 0 to 10. Youths includes youths between 17 and 19 years old. Parents includes parents of youths participating in the experiment. Grandparent includes grandparents of youths participating in the experiment. Second group indicates that the observation corresponds to the individuals participating in the second session of the experiment. 
Table 2 Differences in contribution by group, first vs second group

(1)

(2)

(3)

(4)

First Group Second group Difference p-value diff

\begin{tabular}{ccccc}
\hline \multirow{3}{*}{ Contribution } & \multicolumn{4}{c}{ Panel A: Family } \\
\cline { 2 - 5 } & $\begin{array}{c}6.01 \\
(0.10)\end{array}$ & $\begin{array}{c}7.99 \\
(0.10)\end{array}$ & 1.98 & $(<0.01)$ \\
& & & &
\end{tabular}

Observations

1020

630

\begin{tabular}{cccc}
\hline \multicolumn{4}{c}{ Panel B: Generational } \\
\hline 4.89 & 5.88 & 0.99 & $(<0.01)$ \\
$(0.09)$ & $(0.10)$ & &
\end{tabular}

Observations

1020 630

\begin{tabular}{cccc}
\hline \multicolumn{4}{c}{ Panel C: Random } \\
\hline 4.91 & 5.60 & 0.69 & $(<0.01)$ \\
$(0.10)$ & $(0.10)$ & &
\end{tabular}

Contribution

$(0.10)$

$(0.10)$

Observations

1020

630

Notes: Standard Errors in parenthesis. Contribution measures the contribution in each round to the public good, on a scale from 0 to 10 . Youths includes youths between 17 and 19 years old. Parents includes parents of youths participating in the experiment. Grandparent includes grandparents of youths participating in the experiment. Second group indicates that the observation corresponds to the individuals participating in the second session of the experiment. 
Table 3 RE and FE results for contributions

\begin{tabular}{|c|c|c|c|c|c|c|c|c|}
\hline & (1) & (2) & (3) & (4) & (5) & (6) & (7) & (8) \\
\hline & \multicolumn{2}{|c|}{ All } & \multicolumn{2}{|c|}{ Youths } & \multicolumn{2}{|c|}{ Parents } & \multicolumn{2}{|c|}{ Grandparents } \\
\hline & RE Model & FE Model & RE Model & FE Model & RE Model & FE Model & RE Model & FE Model \\
\hline \multirow[t]{2}{*}{ Family game (ref.: random) } & $1.72 * * *$ & $1.72 * * *$ & $2.46^{* * *}$ & $2.46^{* * *}$ & $1.70 * * *$ & $1.70 * * *$ & $1.01 * * *$ & $1.01 * * *$ \\
\hline & $(0.08)$ & $(0.08)$ & $(0.16)$ & $(0.16)$ & $(0.13)$ & $(0.13)$ & $(0.14)$ & $(0.14)$ \\
\hline \multirow[t]{2}{*}{ Generational game (ref.: random) } & $0.16^{*}$ & $0.16^{*}$ & $0.30^{*}$ & $0.30^{*}$ & -0.07 & -0.07 & $0.23^{*}$ & $0.23^{*}$ \\
\hline & $(0.08)$ & $(0.08)$ & $(0.16)$ & $(0.16)$ & $(0.13)$ & $(0.13)$ & $(0.14)$ & $(0.14)$ \\
\hline \multirow[t]{2}{*}{ Second group in experiment } & $1.23 * * *$ & - & $0.96^{* *}$ & - & $1.44 * *$ & - & $1.22 * * *$ & - \\
\hline & $(0.27)$ & - & $(0.45)$ & - & $(0.58)$ & - & $(0.35)$ & - \\
\hline \multirow[t]{2}{*}{ Round number } & $0.03 * * *$ & $0.03 * * *$ & 0.01 & 0.01 & $0.03 * * *$ & $0.03 * * *$ & $0.04 * * *$ & $0.04 * * *$ \\
\hline & $(0.00)$ & $(0.00)$ & $(0.01)$ & $(0.01)$ & $(0.01)$ & $(0.01)$ & $(0.01)$ & $(0.01)$ \\
\hline \multirow[t]{2}{*}{ Male (ref.: female) } & 0.13 & - & 0.01 & - & 0.68 & - & -0.54 & - \\
\hline & $(0.28)$ & - & $(0.46)$ & - & $(0.59)$ & - & $(0.38)$ & - \\
\hline \multirow[t]{2}{*}{ Age } & 0.09 & - & -3.95 & - & 0.02 & - & 0.08 & - \\
\hline & $(0.08)$ & - & $(5.31)$ & - & $(0.23)$ & - & $(0.07)$ & - \\
\hline \multirow[t]{2}{*}{ Age Squared } & -0.08 & - & 12.24 & - & -0.01 & - & -0.06 & - \\
\hline & $(0.07)$ & - & (15.36) & - & $(0.22)$ & - & $(0.06)$ & - \\
\hline \multirow[t]{2}{*}{ 1st generation (ref.: 3rd generation) } & -0.04 & - & 0 & - & 0 & - & 2.35 & - \\
\hline & $(1.22)$ & - & $(0.00)$ & - & $(0.00)$ & - & $(1.93)$ & - \\
\hline \multirow[t]{2}{*}{ 2nd generation (ref.: 3rd generation) } & -0.07 & - & 0 & - & 3.72 & - & 0 & - \\
\hline & $(1.14)$ & - & $(0.00)$ & - & $(6.22)$ & - & $(0.00)$ & - \\
\hline \multirow[t]{2}{*}{ Constant } & $2.12 *$ & $4.68 * * *$ & 35.19 & $3.94 * * *$ & 0 & $5.16^{* * *}$ & 0 & $4.95 * * *$ \\
\hline & $(1.21)$ & $(0.09)$ & $(45.79)$ & $(0.17)$ & $(0.00)$ & $(0.14)$ & $(0.00)$ & $(0.15)$ \\
\hline Observations & 4,950 & 4,950 & 1,650 & 1,650 & 1,650 & 1,650 & 1,650 & 1,650 \\
\hline R-squared & 0.132 & 0.099 & 0.146 & 0.153 & 0.141 & 0.135 & 0.089 & 0.044 \\
\hline Number of users & 165 & 165 & 55 & 55 & 55 & 55 & 55 & 55 \\
\hline
\end{tabular}

Notes: Robust Standard Errors clustered at the individual level (i.e., users) in parenthesis. Contribution measures the contribution in each round to the public good, on a scale from 0 to 10 . Youths includes youths between 17 and 19 years old. Parents includes parents of youths participating in the experiment. Grandparent includes grandparents of youths participating in the experiment. Second group indicates that the observation corresponds to the individuals participating in the second session of the experiment. The reference group are contributions made in the random assignment treatment during the first session, by females of the $3^{\text {rd }}$ generation (e.g., youths). ${ }^{*}$ Significant at the $90 \%$ level $* *$ Significant at the $95 \%$ level $* * *$ Significant at the $99 \%$. 
Appendix Tutorial (The experiment at the computer)

[First page before going to the login page]

This time from the start of the experiment. Please, stay silent throughout the experiment, turn off your mobile and remember that any element foreign to the experiment is not allowed.

Click here to continue.

[Login page]

Please enter your user name and password on the envelope you have been given.

Username: ...

Password: ...

Click here to continue.

[Next page]

This is an experiment to study how individuals make decisions. Do not think that we expect any particular behaviour. What you do will affect the amount of money you can win. Please, do not talk during the experiment. If you need help, raise your hand and wait to be seen.

Click here to continue.

[Next page]

This experiment consists of three parts. Each part consists, in turn, of an undetermined number of rounds. Each part will take about 5 minutes, but may end earlier. The total duration of the experiment will be about 20 minutes.

You may be gaining different amounts depending on the decisions made in each round by you and the other participants. The gain of each round is expressed in its own currency, the ECU. At the same time, at the end of the experiment, an exchange rate will be set at ECU-euros $(€)$, depending on the number of participants.

The total gain that you can get in this experiment is the sum of the profits earned in all rounds, and converted to $€$, plus a fixed amount of $5 €$ for your participation. This money will be given in cash at the end of the experiment.

Click here to continue.

[Next page] "Decision to be taken in every round"

In this part of the experiment, players are assigned completely at random, peers may be any of the remaining players. They may belong to any generation or may not be related to you (in fact, almost certainly, they are not your family).

In each round, you will have a fixed amount of 10 ECUs. Of those 10 ECUs, you can donate the desired amount into a common fund. The amount you choose not to provide becomes your property, and your benefits accumulate. The other two participants also have 10 ECUs each and, like you, can also contribute to the common fund any amount they wish.

In each round, you have up to 20 seconds to choose how much to contribute to the pool. After 20 seconds, the system will choose for you, but then you can continue to choose without problems in the following rounds. (Do not worry, 20 seconds to choose should be enough).

Once the three participants have chosen, the round ends. At that time, the sum of the amounts that the three participants have contributed to the common fund is multiplied by 1.5 (that is, the program increases the pool 50\%), and the result is divided equally among the three participants, regardless of how much has been invested by each.

Click here to continue. 
[Next page] This is the screen you see during the experiment:

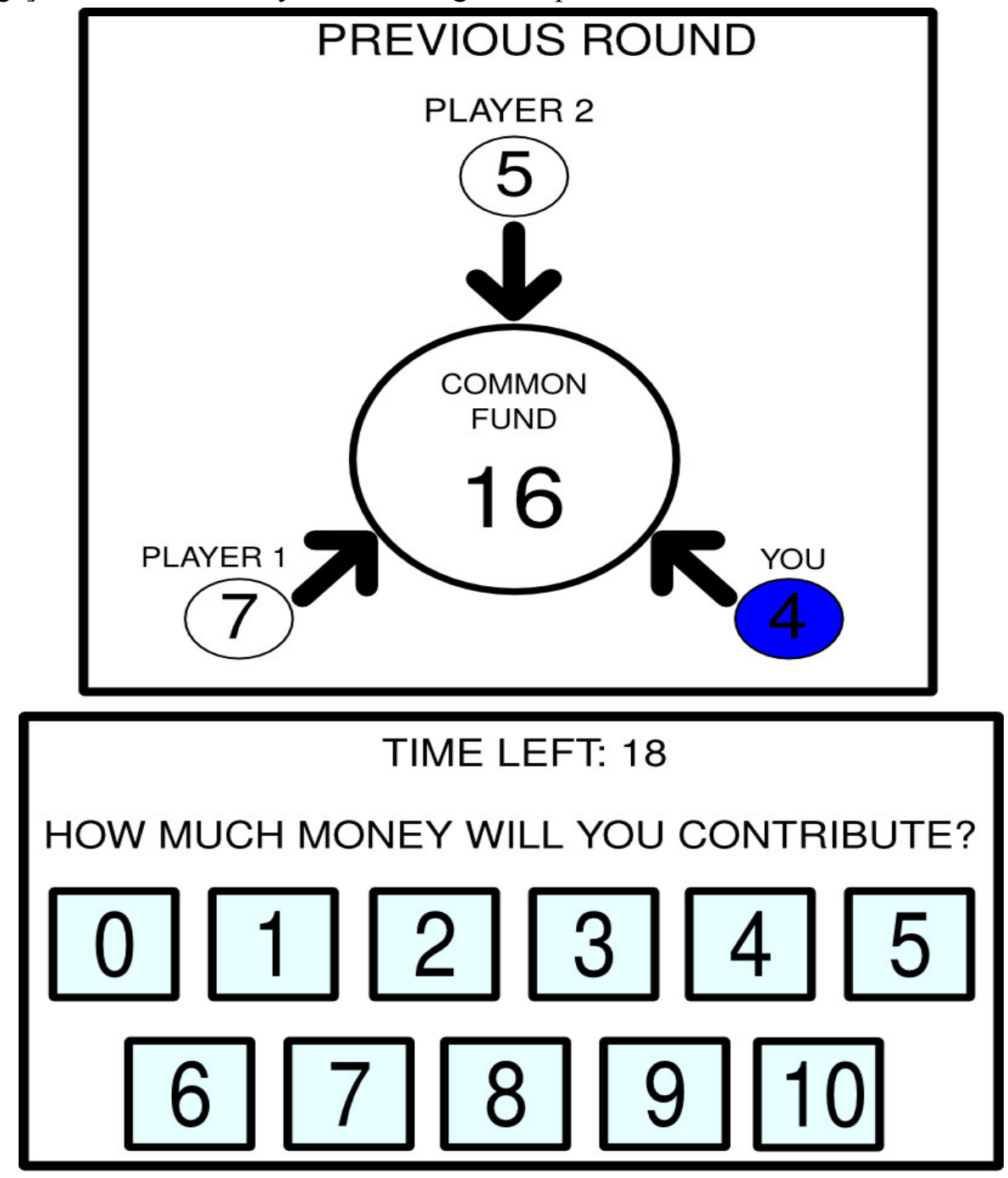

The large central circle represents the common fund. The number inside indicates the total amount the three players contributed to the fund during the previous round. Regarding the three small circles, the blue circle is you and the other two correspond to your companions. The number in each circle indicates how much each contributed to the pool in the previous round. At the bottom of the screen are 11 buttons, from 0 to 10. Simply click on the corresponding amount to invest in the pool.

Click here to continue.

[Next page] "Earnings and repeated round"

Once all participants have made their choice, for that round your total gain is equal to the sum of what you did not contribute plus your share of the common fund, multiplied by 1.5. That amount is accumulated in each round. At the end of the game, your gain is equal to the sum of the profits earned in all rounds.

Remember that each part will be an undetermined number of rounds. In each round, you have up to 20 seconds to choose what amount to deposit in the common fund. After 20 seconds, the system will choose for you, but then you can continue to choose without problems in the following rounds. (Do not worry, 20 seconds to choose should be enough). The round is not over until all participants have chosen.

Click here to continue. 
[Next page] Click here to start Part I of the experiment.

[Part I Begins]

[Game]

Part I ends]

Part I of the experiment is over. Please, keep silent. Begin Part II.

Click here to continue.

[Next page] Tutorial Part II

In this part, players are assigned randomly, and are not necessarily members of your family. You do not know who plays, nor do they know who you are. A difference in the game now is a youth, a parent of a different youth, and a grandfather of another youth are the participants. That is, the three players belong to different generations: youth, father and grandfather, designated on the screen with more words for grandfather / a, lower for the grandson / ae intermediate for the father / mother. However, players are not necessarily members of the same family (in fact, almost certainly, they are not related to each other or to you). Moreover, the experiment is exactly the same as above, any rule changes.

Click here to begin Part II of the experiment.

[Part II Begins]

[Game]

[Part II ends]

Part II of the experiment is over. Please, keep silent. Begin Part III.

Click here to continue.

[Next page] Tutorial Part III

In this part, you will play with two members of your family: his [choose] ffather / mother and grandfather / a \}youth and his / her father / mother \{child and his / her grandson / a \} [end choose ]. The game rules are the same for all participants. Moreover, the experiment is exactly the same as above, with no rule changes.

Click here to start Part III of the experiment.

[Part III Begins]

[Game]

[Part III ends]

Experiment rounds are over. Please, keep silent. Experiment has not finished yet. You must answer the following questions:

Click here to continue.

[Next page] Questions

Did you follow a strategy to decide? BUT

Did you have in mind what the other players contributed to the fund in Part I? BUT

Did you have in mind what the other players contributed to the fund in Part II? BUT

Did you have in mind what their family background contributed to in part III? BUT

[Last page] Experiment is over.

Change ECU-Euro $(€)$ voucher:

$1 E C U=[($ Budget $-\mathrm{n} *$ Attendance Fees $) /$ Total Accumulated Payoff $] €$

You have won: [Payoff (in rounds) + Attendance Fees (5)] $€$

Thank you for your cooperation!! 